\title{
Recent progress in cancer therapy with radiolabeled monoclonal antibodies
}

\author{
"Progress in the use of RAIT for cancer therapy has been slow but steady, involving the reengineering of antibodies (reducing \\ murine component, altering size and clearance properties), and pretargeting methods for improving tumor:blood and \\ tumor:organ ratios are continuing to evolve."
}

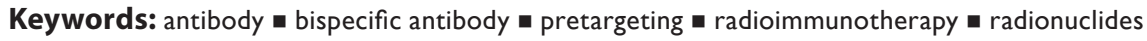

Nearly 60 years ago, the first study showing the feasibility for selective delivery of radionuclides to tumors by antibodies was reported [1], setting the stage for future studies that used antibodies as vehicles for targeting radioactivity, drugs, toxins and other compounds for imaging and therapeutic purposes. Radioimmunotherapy (RAIT), or the antibody-based targeting of therapeutic radionuclides, has been examined clinically for approximately 30 years in a wide range of tumors, but only two agents have received FDA approval in the USA. One is a murine ${ }^{90} \mathrm{Y}$-labeled antiCD20 IgG $\left({ }^{90} \mathrm{Y}\right.$-ibritumomab tiuxetan; Spectrum Pharmaceuticals, Inc., Irvine, CA) given within $4 \mathrm{~h}$ of the patient receiving $250 \mathrm{mg} / \mathrm{m}^{2}$ of rituximab (chimeric anti-CD20 IgG), and the other is a murine ${ }^{131}$ I-labeled anti-CD20 antibody ${ }^{131}$ I-tositumomab; GlaxoSmithKline, Research Triangle Park, NC) given after the infusion of $450 \mathrm{mg}$ unlabeled tositumomab. Both agents in the USA require an imaging study before the therapeutic dose is administered to pre-determine if there is an altered biodistribution that could affect targeting, and in the case of tositumomab, to determine the administered therapeutic ${ }^{131} \mathrm{I}$ activity. Both were approved initially for use in the same indication, specifically patients with relapsed or refractory, low grade, follicular or transformed, non-Hodgkin's lymphoma (NHL). ${ }^{90} \mathrm{Y}$-ibritumomab's approval was based on three separate trials, one a randomized study comparing objective response, duration, and time to progression to rituximab [2]. While the overall and complete response rates favored ${ }^{90} \mathrm{Y}$-ibritumomab (83 and $38 \%$ vs 55 and $18 \%$ for ${ }^{90} Y-i b r i-$ tumomab and rituximab, respectively), there was no difference in response duration and time to progression. Similar response rates and durations were reported for ${ }^{131} \mathrm{I}$-tositumomab in two trials used for its approval [3]. While the more favorable response rates were sufficient to gain regulatory approval, enthusiasm for their use was tempered by their failure to extend the duration of response, particularly because RAIT was more toxic (severe and prolonged myelosuppression), and there were issues with the development of myelodysplatic syndrome and leukemia. However, the incidence of these late complications may have been influenced in a large part by the prior therapies given to most patients $[4,5]$, and there have been a number of subsequent reports indicating that a sizeable portion of the complete responses are long lasting [6,7]. Although neither agent is recommended for first-line therapy, a trial at a single institution showed excellent responses and prolonged remissions in patients with advanced follicular lymphoma [8]. Today, most of the interest for RAIT in lymphoma is as combination with chemotherapy in various settings or as a consolidation treatment [9], providing new opportunities to utilize radiolabeled monoclonal antibodies. In September 2009, ${ }^{90}$ Y-ibritumomab was given supplemental FDA approval to include a second indication as a consolidation treatment for follicular lymphoma patients who achieved partial or complete responses after first-line chemotherapy. It was based on a multicenter, randomized, study of 414 patients who had follicular NHL with a partial or complete responses upon completion of first-line chemotherapy such as single-agent chlorambucil, fludarabine or a fludarabine-containing regimen; a cyclophosphamide-containing combination chemotherapy (e.g., CHOP); or a rituximab-containing combination chemotherapy [10]. Progression-free survival was extended significantly in patients receiving ${ }^{90} \mathrm{Y}-\mathrm{ibritu}-$ momab tiuxetan (median of 28 months) compared with those receiving no further treatment (median of 18 months).

It is not surprising that NHL was RAIT's first approval, since together with leukemias, these cancers are highly sensitive to radiation, and at

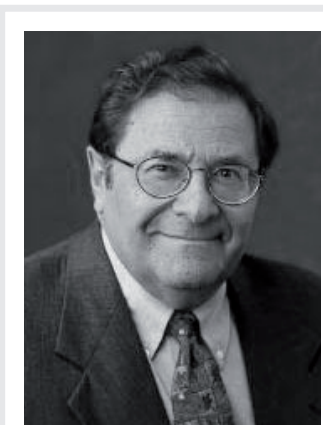

David M Goldenberg

Author for correspondence: Center for Molecular Medicine and Immunology, 300 American Road, Morris Plains, NJ 07950, USA E-mail: dmg.gscancer@att.net

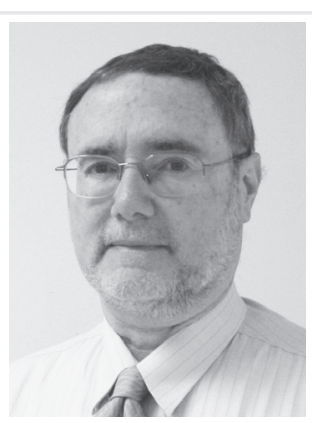

Robert M Sharkey

Center for Molecular Medicine and Immunology, 300 American Road, Morris Plains, NJ 07950, USA 
least in lymphoma, the unlabeled anti-CD20 antibody added to the radioimmunoconjugate contributes to the response [11]. Applying RAIT to leukemias is more difficult, because collateral damage to the bone marrow can cause more severe toxicities, thereby limiting the dose that can be delivered. Unlike the $\beta$-emitters being used for lymphoma therapy, shorter-range $\alpha$-emitters would reduce collateral damage. Clinical trials with bismuth-213 ( $\sim$ h half-life) linked to the humanized anti-CD33 antibody, lintuzumab, have reported significant blast reduction in patients with acute myelogenous leukemia (AML) after partial cytoreductive therapy [12]. An intriguing possibility being explored in animals is the utility of bismuth-213-labeled anti-CD45 antibody for hematopoietic cell transplantation conditioning without being myeloablative [13]. Anti-CD33 and anti-CD45 antibodies labeled with astatine-211 ( $\sim 7 \mathrm{~h}$ half-life) are also being examined for clinical use [14,15]. New cancer therapy trials for leukemia are just beginning with antibodies coupled to the first actinium-225 'nanogenerator' [16]. Unlike most $\alpha$-emitters whose decay is measured in hours, actinium-225 has approximately a 10-day half-life that might be better matched with the biological targeting of a whole IgG. When it decays, it sequentially releases four $\alpha$ particles, amplifying its potential efficacy. However, there are concerns, primarily for bismuth-213 deposition in the kidney, that are being addressed by several approaches to reduce uptake and enhance clearance $[17,18]$.

\section{"Successful treatment of solid tumors with RAIT continues to be a challenge ... Nevertheless, most clinical trials in the USA and Europe are focusing efforts primarily in three areas: regional delivery, minimal disease settings and in combination with chemotherapy."}

Successful treatment of solid tumors with RAIT continues to be a challenge. One agent, an ${ }^{131}$ I-labeled anti-tumor necrosis factor antibody, was approved in China for treating advanced lung cancer either systemically or by injection in the tumor [19]. Nevertheless, most clinical trials in the USA and Europe are focusing efforts primarily in three areas: regional delivery, minimal disease settings and in combination with chemotherapy.

Some of the more common examples of regional therapies include the administration of radiolabeled antibodies in the resection cavity within the brain after surgical removal of the primary lesion, or by intrathecal treatments, both designed primarily to be salvage therapies to remove residual disease and often used in combination with other therapies. A Phase II trial with an ${ }^{131}$ I-labeled murine anti-tenascin antibody in patients with various forms of recurrent brain cancer provided initial encouraging data that this treatment was well tolerated, and together with adjuvant chemotherapy (administered to 58\% of the patients; $35 \%$ received no additional treatments), yielded overall survival data that were favorable compared with other salvage treatment regimens [20]. Kramer et al. reported the results of a trial involving the treatment of 21 patients with recurrent CNS metastases of neuroblastoma, using ${ }^{131}$ I-labeled antibodies targeting GD2 or B7-homologue 3, with most patients also receiving external beam radiation, immunotherapy and chemotherapy. The treatment was tolerated, with myelosuppression being minimized in some individuals by stem cell support. Encouragingly, 17 of the 21 patients were alive 7-74 months after treatment, whereas published results for the median survival in patients not receiving this form of therapy was just 6 months [21]. Intrathecal therapy may also be applied to other cancers [22] .

A second regional therapy is via the intraperitoneal route. One of the largest trials involved the use of a ${ }^{90} Y$-labeled anti-MUC-1 antibody in ovarian cancer patients who had been surgically debulked, but unfortunately did not extend survival or time to relapse [23]. A follow-up analysis of the patients who had relapsed revealed significantly fewer had peritoneal involvement and there was more extraperitoneal involvement in the control arm, suggesting that the peritoneal treatment was effective locally, but it was unable to control metastases outside the treatment zone [24].

RAIT has long been recognized as a treatment that would be best applied in the setting of minimal residual disease $[25,26]$, but most often unimpressive results in Phase I trials in advanced, bulky disease have not provided encouragement to pursue these treatments further. As noted above, even in compartmental treatments, RAIT is primarily being used as a salvage therapy, alone or more often combined with other therapies. Behr et al. [27] and Liersch et al. [28] reported the first evidence in colorectal cancer patients with minimal disease or who had undergone an adjuvant treatment after an R0 resection of hepatic metastases with an ${ }^{131} \mathrm{I}$-anti-CEACAM5 IgG (labetuzumab) may benefit from RAIT. In the adjuvant setting, after a median follow-up 
of 91 months, the median overall survival was 58 months for RAIT-treated patients as compared with 31 months in a contemporaneous group of patients who had not received RAIT after hepatic resection [28]. Thus, for solid tumors, RAIT alone, or combined with other therapies, can potentially provide significant improvements. Recent data from a clinical trial in patients with advanced pancreatic cancer using a humanized ${ }^{90}$ Y-labeled anti-mucin antibody (hPAM4; clivatuzumab tetraxetan) are also providing early evidence that a single RAIT treatment can elicit responses [29]. A follow-up Phase I/II trial in newly diagnosed, inoperable, stage III and IV pancreatic cancer patients examined 3 weekly fractions of this ${ }^{90} \mathrm{Y}$-hPAM4 antibody combined with sub-therapeutic doses of gemcitabine, serving as a radiosensitizer, showed that patients could tolerate nearly twice as much ${ }^{90} \mathrm{Y}$ activity as a single dose in previously treated patients. An interim analysis from this trial indicated disease control (partial response or stable disease) in 21 out of 37 patients (58\%) [30]. These results are encouraging, particularly when considering that most trials in advanced-stage disease have had nominal responses and modest, if any, survival improvement [31]. Preclinical testing of the hPAM4 antibody in human pancreatic cancer xenografts also indicate that therapeutic doses of this or another antibody-SN-38 drug conjugate can be added to RAIT at its maximum tolerated dose to improve responses without increasing toxicities [32], suggesting that this combination of RAIT and chemoimmunotherapy may be a promising new approach.

\section{"Preclinical studies have found pretargeted radionuclides provide superior tumor:nontumor ratios when compared with directly radiolabeled antibodies, and in some systems, tumor uptake can rival that of a directly radiolabeled $\lg G$..."}

While most RAIT trials have used antibodies as IgGs, there is plethora of new recombinant antibodies with improved pharmacokinetics and tumor localization properties that might provide additional improvements in radionuclide targeting [33]. However, directly attaching the radionuclide to the antibody generally leads to dose-limiting hematologic toxicity before a critical dose can be delivered to tumors, since these molecules are cleared from the blood slowly. Smaller forms of antibodies that clear more rapidly reduce the risk for hematologic toxicity, but are generally limited to use with ${ }^{131} \mathrm{I}$ as the therapeutic, since radiometals (e.g., ${ }^{90} \mathrm{Y}$ or ${ }^{177} \mathrm{Lu}$ ) will concentrate to much higher levels in the kidneys than in the tumor [33]. An alternative method, known as pretargeting, couples the radionuclide to a very small compound (peptide) that within a matter of minutes escapes from the vasculature, where it can bind to a specialized antibody conjugate/construct that was pre-localized to the tumor, while the unbound activity is quickly removed from the body by urinary excretion [34]. Preclinical studies have found pretargeted radionuclides provide superior tumor:nontumor ratios when compared with directly radiolabeled antibodies, and in some systems, tumor uptake can rival that of a directly radiolabeled IgG [34,35]. Pretargeting methods in the clinic have most often used streptavidin-biotin or anti-hapten-hapten binding systems. One pretargeting system that was examined in advanced colorectal cancer patients used an antibody that likely bound to EpCam1 (antibody NR-Lu10) conjugated to streptavidin for pretargeting ${ }^{90} \mathrm{Y}$-biotin [36]. Patients received a maximum dose of $110 \mathrm{mCi} / \mathrm{m}^{2}$ of ${ }^{90} \mathrm{Y}$-biotin, which was limited by dose-limiting gastrointestinal toxicity, likely resulting from the antibody conjugate binding to the colon that caused high ${ }^{90} \mathrm{Y}$-biotin uptake in the intestine [36]. A few patients also experienced elevated creatinine, suggesting that the ${ }^{90} Y-$ biotin dose was also likely limited by renal uptake. No significant objective responses were observed, and thus this agent was abandoned. A subsequent pilot clinical study that used a new streptavidin fusion protein targeting TAG-72 did not have the intense intestinal uptake found with the NR-Lu-10 conjugate [37]. In that study, tumor and renal dosimetry data suggested that some patients might have been able to receive as much as 5000 cGy to their tumors at a tolerable dose to the kidneys (e.g., 2000 cGy), but the trial did escalate the ${ }^{90} \mathrm{Y}$-biotin dose [37]. Trials using a somewhat different streptavidin-biotin pretargeting approach also reported promising therapeutic results in brain and ovarian cancers [34]. Since streptavidin is a foreign bacterial protein that is immunogenic, repeated use of this agent is limited.

By contrast, pretargeting systems that use a bispecific anti-tumor-anti-hapten antibody to bind a radiolabeled hapten-peptide potentially can be used on several occasions. Clinical studies with a chemically conjugated anti-CEA Fab' $\times$ anti-DTPA Fab' bispecific antibody and an ${ }^{131}$ I-labeled hapten-peptide have examined 
a number of CEA-producing tumors. A retrospective analysis of patients with medullary thyroid cancer treated with this pretargeting method indicated there was a subset of patients whose survival was improved significantly [38]. More recently, humanized recombinant bispecific antibodies have been developed, with preclinical testing demonstrating them to be excellent platforms for pretargeting a radiolabeled hapten-peptide [39,40]. These recombinant bispecific antibodies have an anti-hapten binding arm that recognizes a unique hapten, HSG (histamine-succinyl-glycine), enabling this system to take advantage of a much wider range of radionuclides than previous anti-DTPA hapten-binding systems. Additionally, these bispecific antibodies have the capacity to bind divalently to the tumor antigen, which can further enhance the capture of the radiolabeled hapten-peptide [39]. Used in imaging applications, this bispecific antibody-pretargeting system has been found to be more sensitive and specific than ${ }^{18} \mathrm{~F}$-fluorodeoxyglucose for targeting colorectal cancer xenografts, using hapten-peptides capable of being radiolabeled with ${ }^{124} \mathrm{I},{ }^{68} \mathrm{Ga}$, or even ${ }^{18} \mathrm{~F}[40-43]$. Therapy studies in xenograft models for colorectal and pancreatic cancers, and also NHL, have shown improved responses with less toxicity for pretargeting over direct targeting. Clinical trials are now just beginning with this new method and agents [40].

\section{Conclusion \& future perspective}

Progress in the use of RAIT for cancer therapy has been slow but steady, involving the reengineering of antibodies (reducing murine component, altering size and clearance properties), and pretargeting methods for improving tumor:blood and tumor:organ ratios are continuing to evolve. Radiochemistry has improved, allowing for more diversity in targeting therapeutic $\beta$-, $\alpha$ - and Auger electron-emitting radionuclides. In lymphoma and in solid tumors clinical trials are expanding in different clinical settings. Also being studied is the possible improvement in cancer targeting and binding with bispecific antibodies, where more than one antigen or more than one epitope of an antigen is targeted for improved accretion. These prospects stimulate continued enthusiasm for the pursuit and study of how therapeutic radionuclides can be localized to cancer cells selectively.

\section{Financial \& competing interests disclosure} David $M$ Goldenberg has financial interests in Immunomedics, Inc. The authors have no other relevant affiliations or financial involvement with any organization or entity with a financial interest in or financial conflict with the subject matter or materials discussed in the manuscript apart from those disclosed.

No writing assistance was utilized in the production of this manuscript.

\section{Bibliography}

1 Goldenberg DM, Sharkey RM. Radioactive antibodies: a historical review of selective targeting and treatment of cancer. Hosp. Pract. (Minneap.) 38(5), 82-93 (2010).

$2 Z_{\text {evalin }}^{\circledR}$, package insert. Spectrum Pharmaceuticals, Irvine, CA, USA.

3 Bexxar $^{\circledast}$, package insert. GlaxoSmithKline, Research Triangle Park, NC, USA.

4 Bennett JM, Kaminski MS, Leonard JP et al. Assessment of treatment-related myelodysplastic syndromes and acute myeloid leukemia in patients with non-Hodgkin lymphoma treated with tositumomab and iodine $\mathrm{I}^{131}$ tositumomab. Blood 105(12), 4576-4582 (2005).

5 Czuczman MS, Emmanouilides C, Darif $\mathrm{M}$ et al. Treatment-related myelodysplastic syndrome and acute myelogenous leukemia in patients treated with ibritumomab tiuxetan radioimmunotherapy. J. Clin. Oncol. 25(27), 4285-4292 (2007).
6 Fisher RI, Kaminski MS, Wahl RL et al. Tositumomab and iodine-131 tositumomab produces durable complete remissions in a subset of heavily pretreated patients with low-grade and transformed non-Hodgkin's lymphomas. J. Clin. Oncol. 23(30), 7565-7573 (2005).

7 Witzig TE, Molina A, Gordon LI et al. Long-term responses in patients with recurring or refractory B-cell non-Hodgkin's lymphoma treated with yttrium 90 ibritumomab tiuxetan. Cancer 109(9), 1804-1810 (2007).

8 Kaminski MS, Tuck M, Estes J et al. ${ }^{131}$ I-tositumomab therapy as initial treatment for follicular lymphoma. N. Engl. J. Med. 352(5), 441-449 (2005).

9 Ahmed S, Winter JN, Gordon LI, Evens AM. Radioimmunotherapy for the treatment of non-Hodgkin lymphoma: current status and future applications. Leuk. Lymphoma 51(7), 1163-1177 (2010).

10 Morschhauser F, Radford J, Van Hoof A et al. Phase III trial of consolidation therapy with yttrium-90-ibritumomab tiuxetan compared with no additional therapy after first remission in advanced follicular lymphoma. J. Clin. Oncol. 26(32), 5156-5164 (2008).

11 Sharkey RM, Press OW, Goldenberg DM. A re-examination of radioimmunotherapy in the treatment of non-Hodgkin lymphoma: prospects for dual-targeted antibody/ radioantibody therapy. Blood 113(17), 3891-3895 (2009).

12 Rosenblat TL, McDevitt MR, Mulford DA et al. Sequential cytarabine and $\alpha$-particle immunotherapy with bismuth-213lintuzumab (HuM195) for acute myeloid leukemia. Clin. Cancer Res. 16(21), 5303-5311 (2010).

13 Nakamae H, Kerbauy FR, Wilbur DS et al. Pilot study of a ${ }^{213}$ bismuth-labeled anti-CD 45 $\mathrm{mAb}$ as a novel nonmyeloablative conditioning for DLA-haploidentical littermate hematopoietic transplantation. Transplantation 89(11), 1336-1340 (2010).

14 Nakamae H, Wilbur DS, Hamlin DK et al. Biodistributions, myelosuppression, and toxicities in mice treated with an anti-CD45 
antibody labeled with the $\alpha$-emitting radionuclides bismuth-213 or astatine- 211 . Cancer Res. 69 (6), 2408-2415 (2009).

15 Petrich T, Korkmaz Z, Krull D, Fromke C, Meyer GJ, Knapp WH. In vitro experimental ${ }^{211}$ At-anti-CD33 antibody therapy of leukaemia cells overcomes cellular resistance seen in vivo against gemtuzumab ozogamicin. Eur. J. Nucl. Med. Mol. Imaging 37(5), 851-861 (2010).

16 Miederer M, Scheinberg DA, McDevitt MR. Realizing the potential of the actinium-225 radionuclide generator in targeted $\alpha$ particle therapy applications. Adv. Drug Deliv. Rev. 60(12), 1371-1382 (2008).

17 Schwartz J, Jaggi JS, O’Donoghue JA et al. Renal uptake of bismuth-213 and its contribution to kidney radiation dose following administration of actinium-225labeled antibody. Phys. Med. Biol. 56(3), 721-733 (2011)

18 Jaggi JS, Seshan SV, McDevitt MR, Sgouros G, Hyjek E, Scheinberg DA. Mitigation of radiation nephropathy after internal $\alpha$-particle irradiation of kidneys. Int. J. Radiat. Oncol. Biol. Phys. 64(5), 1503-1512 (2006).

19 Chen S, Yu L, Jiang C et al. Pivotal study of iodine-131-labeled chimeric tumor necrosis treatment radioimmunotherapy in patients with advanced lung cancer. J. Clin. Oncol. 23(7), 1538-1547 (2005).

20 Reardon DA, Zalutsky MR, Bigner DD. Antitenascin- $\mathrm{C}$ monoclonal antibody radioimmunotherapy for malignant glioma patients. Expert Rev. Anticancer Ther. 7(5), 675-687 (2007).

21 Kramer K, Kushner BH, Modak S et al. Compartmental intrathecal radioimmunotherapy: results for treatment for metastatic CNS neuroblastoma. J. Neurooncol. 97(3), 409-418 (2010).

22 Boskovitz A, McLendon RE, Okamura T, Sampson JH, Bigner DD, Zalutsky MR. Treatment of HER2-positive breast carcinomatous meningitis with intrathecal administration of $\alpha$-particle-emitting ${ }^{211}$ At-labeled trastuzumab. Nucl. Med. Biol. 36(6), 659-669 (2009).

23 Verheijen RH, Massuger LF, Benigno BB et al. Phase III trial of intraperitoneal therapy with yttrium-90-labeled HMFG1 murine monoclonal antibody in patients with epithelial ovarian cancer after a surgically defined complete remission. J. Clin. Oncol. 24(4), 571-578 (2006)

24 Oei AL, Verheijen RH, Seiden MV et al. Decreased intraperitoneal disease recurrence in epithelial ovarian cancer patients receiving intraperitoneal consolidation treatment with yttrium-90-labeled murine HMFG1 without improvement in overall survival. Int. J. Cancer 120(12), 2710-2714 (2007).

25 Boerman OC, Sharkey RM, Blumenthal RD, Aninipot RL, Goldenberg DM. The presence of a concomitant bulky tumor can decrease the uptake and therapeutic efficacy of radiolabeled antibodies in small tumors. Int. J. Cancer 51(3), 470-475 (1992).

26 Sharkey RM, Pykett MJ, Siegel JA, Alger EA, Primus FJ, Goldenberg DM.

Radioimmunotherapy of the GW-39 human colonic tumor xenograft with ${ }^{131}$-labeled murine monoclonal antibody to carcinoembryonic antigen. Cancer Res. 47(21), 5672-5677 (1987).

27 Behr TM, Liersch T, Greiner-Bechert L et al. Radioimmunotherapy of small-volume disease of metastatic colorectal cancer. Cancer 94(4 Suppl.), 1373-1381 (2002).

28 Liersch T, Meller J, Bittrich M, Kulle B, Becker H, Goldenberg DM. Update of carcinoembryonic antigen radioimmunotherapy with ${ }^{131}$ I-labetuzumab after salvage resection of colorectal liver metastases: comparison of outcome to a contemporaneous control group. Ann. Surg. Oncol. 14(9), 2577-2590 (2007).

29 Gulec AA, Cohen SJ, Pennington KL et al. Treatment of advanced pancreatic carcinoma with ${ }^{90} Y$-clivatuzumab tetraxetan (humanized anti-pancreatic mucin antibody, hPAM4): a Phase I dose escalation trial. Clin. Cancer Res. doi: 10.1158/1078-0432.CCR-10-2579 (2011) (Epub ahead of print).

30 Ocean AJ, Guarino MJ, Pennington KL et al. Therapeutic effects of fractionated radioimmunotherapy (RAIT) with clivatuzumab tetraxetan combined with low-dose gemcitabine (Gem) in advanced pancreatic cancer (APC). J. Clin. Oncol. 28, 15s (2010) (Abstract 4115).

31 Sharkey RM, Goldenberg DM. Perspectives on cancer therapy with radiolabeled monoclonal antibodies. J. Nuc. Med. 46(Suppl. 1), 115S-127S (2005).

32 Sharkey RM, Karacay H, Govindan SV, Goldenberg DM. Combination radioimmunotherapy and chemoimmunotherapy involving different or the same targets improves therapy of human pancreatic carcinoma xenograft models. Mol. Cancer. Ther. 10, 1072-1081 (2011)

33 Sharkey RM, Goldenberg DM. Novel radioimmunopharmaceuticals for cancer imaging and therapy. Curr. Opin. Investig. Drugs 9(12), 1302-1316 (2008).
34 Goldenberg DM, Sharkey RM, Paganelli G, Barbet J, Chatal JF. Antibody pretargeting advances cancer radioimmunodetection and radioimmunotherapy. J. Clin. Oncol. 24(5), 823-834 (2006).

35 Goldenberg DM, Chatal JF, Barbet J, Boerman O, Sharkey RM. Cancer imaging and therapy with bispecific antibody pretargeting. Update Cancer Ther. 2(1), 19-31 (2007).

36 Knox SJ, Goris ML, Tempero M et al. Phase II trial of yttrium-90-DOTA-biotin pretargeted by NR-LU-10 antibody/ streptavidin in patients with metastatic colon cancer. Clin. Cancer Res. 6(2), 406-414 (2000).

37 Shen S, Forero A, LoBuglio AF et al. Patient-specific dosimetry of pretargeted radioimmunotherapy using CC49 fusion protein in patients with gastrointestinal malignancies. J. Nucl. Med. 46(4), 642-651 (2005).

38 Chatal JF, Campion L, Kraeber-Bodere F et al. Survival improvement in patients with medullary thyroid carcinoma who undergo pretargeted anti-carcinoembryonicantigen radioimmunotherapy: a collaborative study with the French Endocrine Tumor Group. J. Clin. Oncol. 24(11), 1705-1711 (2006).

39 Sharkey RM, Rossi EA, Chang $\mathrm{CH}$, Goldenberg DM. Improved cancer therapy and molecular imaging with multivalent, multispecific antibodies. Cancer Biother. Radiopharm. 25(1), 1-12 (2010).

40 Sharkey RM, Rossi EA, McBride WJ, Chang $\mathrm{CH}$, Goldenberg DM. Recombinant bispecific monoclonal antibodies prepared by the dock-and-lock strategy for pretargeted radioimmunotherapy. Semin. Nucl. Med. 40(3), 190-203 (2010).

41 Karacay H, Sharkey RM, McBride WJ, Rossi EA, Chen C-H, Goldenberg DM. Optimization of hapten-peptide labeling for bispecific antibody pretargeted ImmunoPET using generator-produced ${ }^{68} \mathrm{Ga}$. J. Nucl. Med. 52(4), 555-559 (2011).

42 Schoffelen R, Sharkey RM, Goldenberg DM et al. Pretargeted immuno-positron emission tomography imaging of carcinoembryonic antigen-expressing tumors with a bispecific antibody and a ${ }^{68} \mathrm{Ga}$ - and ${ }^{18} \mathrm{~F}$-labeled hapten peptide in mice with human tumor xenografts. Mol. Cancer. Ther. 9(4), 1019-1027 (2010).

43 McBride WJ, D'Souza CA, Sharkey RM et al. Improved ${ }^{18} \mathrm{~F}$ labeling of peptides with a fluoride-aluminum-chelate complex. Bioconjug. Chem. 21(7), 1331-1340 (2010). 